\title{
STREBLIDAE (DIPTERA, HIPPOBOSCOIDEA) EMMORCEGOS (CHIROPTERA, PHYLLOSTOMIDAE) NO NORDESTE DO RIO GRANDE DO SUL, BRASIL
}

\author{
Gustavo Graciolli ${ }^{1}$ \\ Ana Maria Rui ${ }^{2}$
}

\begin{abstract}
STREBLIDAE (DIPTERA, HIPPOBOSCOIDEA) ON BATS (CHIROPTERA, PHYLLOSTOMIDAE) IN THE NORTHEAST OF RIO GRANDE DO SUL, BRAZIL. A survey of the Streblidae batflies on the phyllostomid bats was conducted in the northeastern Rio Grande do Sul State, Brazil, during 1997. Hundred thirty three streblids were collected on 44 parasited hosts. Eleven species of batflies (Trichobius dugesii Townsend, 1891, T. tiptoni Wenzel, 1976, Trichobius sp., Paratrichobius longicrus (Miranda Ribeiro, 1907), Megistopoda aranea (Coquillett, 1899), M. proxima (Séguy, 1926), Exastinion clovisi (Pessoa \& Guimarães, 1936), Paraeuctenodes longipes Pessoa \& Guimarães, 1936, Anastrebla modestini Wenzel, 1966, A. caudiferae Wenzel, 1976 and Metelasmus pseudopterus Coquillett, 1907) were found on six species of phyllostomid bats (Artibeus lituratus (Olfers, 1818), A. fimbriatus Gray, 1838, Sturnira lilium (E. Geoffroy, 1810), Glossophaga soricina (Pallas, 1766), Anoura caudifera (E. Geoffroy, 1818) and A. geoffroyi Gray, 1838). All records are new for the Rio Grande do Sul and Anastrebla caudiferae is firstly recorded in Brazil. Differences in the batflies community composition in Artibeus fimbriatus and A. lituratus are discussed.
\end{abstract}

KEYWORDS. Streblidae, Hippoboscoidea, ectoparasites, Phyllostomidae, Rio Grande do Sul.

\section{INTRODUÇÃO}

Stréblidas são hematófagos com viviparidade adenotrófica, ectoparasitos exclusivos de morcegos, encontrados, principalmente, na Região Neotropical. Dos 31 gêneros aceitos, 25 ocorrem no Novo Mundo e cerca de 66\% das espécies conhecidas são americanas (GUERRERo, 1993). Levantamentos taxonômicos foram realizados no México (Guerrero \& Morales-Malacara, 1996), em Trinidad (Jobling, 1949), no Panamá (Wenzel et al., 1966), Colômbia (Bequeart, 1940; Marinkelle \& Grose, 1981) e Venezuela (Bequeart, 1942;

1. Departamento de Zoologia, Universidade Federal do Paraná, Caixa Postal 19020, CEP 81531-970, Curitiba, Paraná, Brasil (mingau@ bio.ufpr.br).

2. Departamento de Ecologia, Universidade de Brasília, Campus Universitário, Asa Norte, CEP 70919-970, Brasília, Distrito Federal, Brasil (anarui@unb.br) 
WenZel, 1976). No Brasil, foram registradas em torno de 53 espécies, número inferior ao encontrado no Panamá e na Venezuela, por Pessoa \& Guimarães (1940) no Mato Grosso, WhitAKer \& Mumford (1977) e Komeno \& Linhares (1999) em Minas Gerais e CoImbra et al. (1984) no Distrito Federal.

O objetivo foi realizar um levantamento das espécies de estréblidas parasitas de morcegos filostomídeos no norte da Planície Costeira do Estado do Rio Grande do Sul, Região Sul do Brasil.

\section{MATERIAL E MÉTODOS}

O trabalho foi realizado em Dom Pedro de Alcântara $\left(29^{\circ} 18^{\prime} \mathrm{S}, 49^{\circ} 44^{\prime} \mathrm{W}\right)$, no extremo norte da Planície Costeira do Rio Grande do Sul, Brasil. A região é de domínio da Floresta Ombrófila Densa (IBGE, 1986) e atualmente restam apenas fragmentos de florestas muitas vezes alterados, que são cercados por lavouras, plantações de bananeiras e pastagens para o gado.

As coletas dos morcegos foram realizadas durante o ano de 1997, em fragmentos de mata, com redes de neblina de $12 \times 2,5 \mathrm{~m}$ armadas em trilhas e em uma furna à beira da BR 101, km 13/14. As redes eram armadas ao anoitecer e desarmadas ao amanhecer, sendo revisadas periodicamente ao longo da noite. Os morcegos capturados foram identificados, sexados, pesados, tiveram os antebraços medidos e, a seguir, foram libertados.

Os estréblidas foram removidos do corpo do hospedeiro segundo as técnicas indicadas por WHITAKER (1988) e fixados em álcool etílico 70\%. As amostras foram individualizadas por hospedeiro e devidamente etiquetadas. A identificação das espécies de Streblidae foi realizada utilizando as chaves de GuerRero (1993, 1994, 1995a, b, 1996) e comparando com material depositado no Museu de Zoologia da Universidade de São Paulo, São Paulo. O ordenamento das espécies de Streblidae segue a proposta taxonômica de Guerrero (1997). Os Streblidae estão depositados na Coleção de Entomologia Padre Jesus Santiago Moure (DZUP), Departamento de Zoologia, Universidade Federal do Paraná, Curitiba (PR).

Índices ecológicos foram utilizados para discutir a composição da comunidade de ectoparasitas, apenas nas espécies de hospedeiros mais abundantes (Artibeus fimbriatus Gray, 1838 e A. lituratus (Olfers, 1818)). Os Índices de Prevalência ( ${ }^{\circ}$ de hospedeiros infestados por um táxon de parasita $/ \mathrm{n}^{\circ}$ de hospedeiros examinados) e Abundância Média ( $\mathrm{n}^{\circ}$ total de indivíduos de uma espécie de parasita amostrada em uma espécie de hospedeiro $/ \mathrm{n}^{\circ}$ total de hospedeiros daquela espécie examinados) foram utilizados conforme Bush et al. (1997).

\section{RESULTADOS E DISCUSSÃO}

Foram coletados 133 indivíduos de estréblidas sobre 44 filostomídeos parasitados. Onze espécies de Streblidae são registradas para o Rio Grande do Sul, relacionadas a 6 espécies de filostomídeos (tab. I). Anastrebla caudiferae Wenzel, 1976 é registro novo para o Brasil.

\section{TRICHOBIINAE Trichobius dugesii Townsend, 1891}

Material examinado. BRASIL. Rio Grande do Sul: Dom Pedro de Alcântara, sobre Glossophaga soricina (Pallas, 1766): O', Graciolli \& Rui col., 19.VI.1997.

Comentário. Guimarães (1941) cita a ocorrência de T. dugesii sobre Glossophaga soricina em São Paulo, sobre Phyllostomus hastatus (Pallas, 1767) em Santa Catarina e sobre Carollia perspicillata (L., 1758) no Amazonas. 


\section{Trichobius tiptoni Wenzel, 1976}

Material examinado. BRASIL. Rio Grande do Sul: Dom Pedro de Alcântara, sobre Anoura caudifera (E. Geoffroy, 1818): 3 \%, 3 9, Graciolli \& Rui col., 24.IV.1997; Furna BR 101, km 13/14, sobre A. geoffroyi Gray, 1838: 2 \%, Grillo col., 6,7.IX.1997; sobre A. caudifera: 5 o', ९, Grillo col., 7,8.IX.1997.

Comentário. Para Minas Gerais, Komeno \& LinHAREs (1999) citam a ocorrência de Trichobius tiptoni sobre Anoura caudifera. Este é o primeiro registro de T. tiptoni parasitando A. geoffroyi; no entanto, as duas espécies de Anoura estavam coexistindo no mesmo abrigo e talvez essa associação possa ser uma contaminação.

Nota. Uma fêmea de Trichobius sp., pertencente ao grupo phyllostomae, foi coletada em Dom Pedro de Alcântara, sobre Sturnira lilium (E. Geoffroy, 1810): Rui col., 20.II.1997. Está espécie é semelhante à T. brennani Wenzel, 1966.

\section{Paratrichobius longicrus (Miranda Ribeiro, 1907)}

Material examinado. BRASIL. Rio Grande do Sul: Dom Pedro de Alcântara, sobre Artibeus fimbriatus: 2 , , Graciolli \& Rui col., 24.IV.1997; $\sigma^{-6}$, Graciolli \& Rui col., 09.V.1997; sobre $A$. lituratus: o', 2 9, Graciolli \& Rui col., 24, 25.IV.1997; 9 o', 4 9, Graciolli \& Rui col., 09, 10.V.1997; ९, Graciolli \& Rui col., 20.VI.1997; O', Y, Graciolli \& Rui col., 25.VII.1997; ९, Graciolli \& Rui col., sem data.

Comentário. Paratrichobius longicrus é parasita primário de Artibeus lituratus; no entanto, pode ser encontrada parasitando outras espécies de Artibeus (A. aztecus Andersen, 1906; A. concolor Peters, 1865; A. jamaicensis Leach, 1821) (GUERRERO, 1994; WeNZEL et al., 1966). No Brasil, P. longicrus foi encontrada sobre Artibeus jamaicensis no Rio de Janeiro (Miranda Ribeiro, 1907) e em Goiás (PessoA \& Guimarães, 1936); A. lituratus em Minas Gerais (WhitaKer \& Mumford, 1977) e no Distrito Federal (CoImbra et al., 1984); Platyrrhinus lineatus (E. Geoffroy, 1810) em Minas Gerais (KoMENo \& LinHAREs, 1999) e Carollia perspicillata em São Paulo (PessoA \& Guimarães, 1936).

\section{Megistopoda aranea (Coquillett, 1899)}

Material examinado. BRASIL. Rio Grande do Sul: Dom Pedro de Alcântara, sobre Artibeus

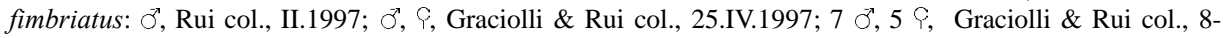
10.V.1997; sobre A. lituratus: Y, Graciolli \& Rui col., II.1997; 2 o', Graciolli \& Rui col., 09.V.1997.

Comentário. Megistopoda aranea é encontrada principalmente sobre espécies de Artibeus, sendo considerada como parasita primário de A. jamaicensis, apesar de já ter sido coletada sobre outros morcegos filostomídeos. Autino et al. (1991) e BARQuEz et al. (1991) citaram a ocorrência sobre A. planirostris (Spix, 1823) no norte da Argentina e Komeno \& Linhares (1999) para Minas Gerais. Autino et al. (1998), no Parque Nacional Iguazú, Jujuy, Argentina, registraram pela primeira vez a associação entre $M$. aranea e $A$. fimbriatus. No Brasil, M. aranea já foi registrada no Pará, São Paulo e Santa Catarina (WENZEL, 1970).

\section{Megistopoda proxima (Séguy, 1926)}

Material examinado. BRASIL. Rio Grande do Sul: Dom Pedro de Alcântara, sobre Sturnira

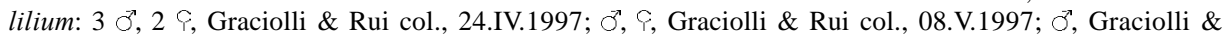
Rui col., 25.VII.1997. 
Comentário. Parasita principalmente espécies de Sturnira em toda distribuição geográfica do gênero. No Brasil, Wenzel et al. (1966) registraram a ocorrência no Pará e em Santa Catarina. Em Minas Gerais, Whitaker \& Mumford (1977) e Komeno \& Linhares (1999) registraram Megistopoda proxima sobre Sturnira lilium; no entanto, CoIMBRA et al. (1984) citaram a ocorrência sobre Carollia perspicillata no Distrito Federal. GuERRERO (1994) examinou exemplares provenientes do Uruguai, coletados sobre Oxymicterus rufus nasutus (Waterhouse, 1837), um roedor.

\section{Exastinion clovisi (Pessoa \& Guimarães, 1936)}

Material examinado. BRASIL. Rio Grande do Sul: Dom Pedro de Alcântara, sobre Anoura caudifera: 2 \%, 2 , Graciolli \& Rui col., 20.VI.1997; Furna BR 101, km 13/14, sobre Anoura sp.: 10 on, 6 9, Graciolli, Rui \& Grillo col., 19.VI.1997; sobre A. geoffroyi: 2 o, 5 9, Grillo col., 6,7.IX.1997; sobre A. caudifera:, , Grillo col., 08.IX.1997.

Comentário. Parasita principalmente espécies de Anoura. Há registro de sua ocorrência no México, Guatemala, Panamá, Trinidad, Venezuela e Brasil (Wenzel, 1970; Guerrero \& Morales-Malacara, 1996). No Brasil, já foi encontrada em Minas Gerais (Whitaker \& Mumford, 1977; Komeno \& Linhares, 1999), Mato Grosso e São Paulo (Pessoa \& Guimarães, 1936; 1940) sobre A. geoffroyi.

\section{STREBLINAE \\ Paraeuctenodes longipes Pessoa \& Guimarães, 1936}

Material examinado. BRASIL. Rio Grande do Sul: Dom Pedro de Alcântara, sobre Glossophaga soricina: 4 O', Y, Graciolli \& Rui col., 19.VI.1997.

Comentário. Durante o manuseio do hospedeiro, foi observado que os exemplares de $P$. longipes realizavam pequenos vôos elípticos, abandonando e logo retornando ao hospedeiro. No Brasil, esta espécie somente tinha sido coletada em São Paulo (PESSOA \& GuIMARÃES, 1936).

\section{Anastrebla modestini Wenzel, 1966}

Material examinado. BRASIL. Rio Grande do Sul: Dom Pedro de Alcântara, sobre Anoura geoffroyi: $2 \sigma^{\circ}$, Graciolli \& Rui col., 20.VI.1997; Furna BR 101, km 13/14, sobre Anoura sp.: 7 \%, 9, Graciolli, Rui, \& Grillo col., 19.VI.1997; sobre A. geoffroyi: 3 o', 2 \&, Grillo col., 6,7.IX.1997.

Comentário. WhitAKer \& MuMFord (1977) encontraram esta espécie parasitando $A$. geoffroyi em Minas Gerais.

\section{Anastrebla caudiferae Wenzel, 1976}

Material examinado. BRASIL. Rio Grande do Sul: Dom Pedro de Alcântara, sobre Anoura caudifera: 3 o, 3 9, Graciolli \& Rui col., 24.IV.1997; Furna BR 101, km 13/14, sobre Anoura sp.: $\sigma^{\prime}$, Graciolli, Rui \& Grillo col., 19.VI.1997; sobre A. caudifera: 3 o', Y, Grillo col., 08.IX.1997.

Comentário. Primeiro registro de ocorrência desta espécie para o Brasil; anteriormente havia sido encontrada apenas na Venezuela (GuERRERo, 1996). 


\section{Metelasmus pseudopterus Coquillett, 1907}

Material examinado. BRASIL. Rio Grande do Sul: Dom Pedro de Alcântara, sobre Artibeus

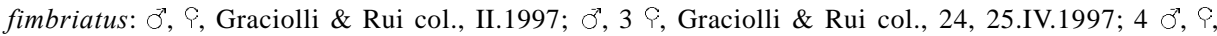
Graciolli \& Rui col., 09.V.1997; đ̈, Graciolli \& Rui col., 25.VII.1997.

Comentário. Parasita primário de Artibeus jamaicensis. BARQUEZ et al. (1991) encontraram-na parasitando A planirostris no noroeste argentino. No Brasil, já foi encontrada nos estados do Pará, Santa Catarina (Wenzel, 1970) e São Paulo (PessoA \& Galvão, 1936). Este é o primeiro registro de M. pseudopterus parasitando A. fimbriatus.

Foram coletadas duas espécies de estréblidas em Artibeus lituratus, Sturnira lilium e Glossophaga soricina e três espécies em Artibeus fimbriatus, Anoura caudifera e Anoura geoffroyi. Observou-se que os estréblidas estudados estão restritos a uma espécie ou gênero de morcego filostomídeo. Das 11 espécies de ectoparasitas coletadas, 7 ocorreram em apenas uma espécie de filostomídeo, Trichobius tiptoni e Exastinion clovisi ocorreram em Anoura caudifera e A. geoffroyi e Paratrichobius longicrus e Megistopoda aranea ocorreram em Artibeus lituratus e A. fimbriatus (tab. I). Esta especificidade do ectoparasita em relação ao hospedeiro já havia sido constatada por Ross (1961) e WENZEL et al. (1966).

A proporção sexual dos estréblidas foi de 1 macho: 0,61 fêmea (tab. II). MARSHALL (1981), analisando estatisticamente a coleção realizada por Wenzel (1976) na Venezuela, encontrou diferenças significativas para o maior número de machos em Megistopoda aranea e Metelasmus pseudopterus e não houve diferenças significativas para Exastinion clovisi e Paratrichobius longicrus. Comentou que em espécies braquípteras e ápteras normalmente há uma proporção sexual mais equilibrada do que em espécies aladas. Este fato foi constatado para E. clovisi em Dom Pedro de Alcântara.

Tabela I. Espécies de Streblidae e seus respectivos hospedeiros, morcegos da família Phyllostomidae, coletados em Dom Pedro de Alcântara, Rio Grande do Sul, Brasil, durante 1997.

\begin{tabular}{|c|c|c|c|c|c|c|}
\hline \multirow[t]{2}{*}{ Streblidae } & \multicolumn{6}{|c|}{ Phyllostomidae } \\
\hline & $\begin{array}{l}\text { Artibeus } \\
\text { lituratus }\end{array}$ & $\begin{array}{c}\text { Artibeus } \\
\text { fimbriatus }\end{array}$ & $\begin{array}{c}\text { Sturnira } \\
\text { lilium }\end{array}$ & $\begin{array}{l}\text { Glossophaga } \\
\text { soricina }\end{array}$ & $\begin{array}{l}\text { Anoura } \\
\text { caudifera }\end{array}$ & $\begin{array}{l}\text { Anoura } \\
\text { geoffroyi }\end{array}$ \\
\hline \multicolumn{7}{|l|}{ Trichobiinae } \\
\hline Trichobius dugesii & & & & $\mathrm{X}$ & & \\
\hline T. tiptoni & & & & & $\mathrm{X}$ & $\mathrm{X}$ \\
\hline Trichobius sp. & & & $\mathrm{X}$ & & & \\
\hline Paratrichobius longicrus & $\mathrm{X}$ & $\mathrm{X}$ & & & & \\
\hline Megistopoda aranea & $\mathrm{X}$ & $X$ & & & & \\
\hline M. proxima & & & $\mathrm{X}$ & & & \\
\hline Exastinion clovisi & & & & & $X$ & $X$ \\
\hline \multicolumn{7}{|l|}{ Streblinae } \\
\hline Paraeuctenodes longipes & & & & $X$ & & \\
\hline Anastrebla modestini & & & & & & $X$ \\
\hline A. caudiferae & & & & & $\mathrm{X}$ & \\
\hline Metelasmus pseudopterus & & $\mathrm{X}$ & & & & \\
\hline Total & 2 & 3 & 2 & 2 & 3 & 3 \\
\hline
\end{tabular}


Durante o período de amostragem, foram coletados 23 exemplares de Artibeus fimbriatus, dos quais 13 (56,5\%) estavam parasitados e 37 A. lituratus, destes 14 (37,8\%) parasitados. A. fimbriatus foi parasitado por três espécies de estréblidas, onde em um indivíduo estavam coexistindo Paratrichobius longicrus e Megistopoda aranea e em dois outros, M. aranea e Metelasmus pseudopterus (tab. III). Em A. lituratus, foram encontradas duas espécies, $P$. longicrus e $M$. aranea e em apenas dois indivíduos estas estavam coexistindo (tab. III).

Tabela II. Número de machos e fêmeas e proporção sexual das espécies de Streblidae em Dom Pedro de Alcântara, Rio Grande do Sul, Brasil, durante 1997.

\begin{tabular}{lccc}
\hline \multicolumn{1}{c}{ Streblidae } & Machos & Fêmeas & Proporção sexual \\
\hline Trichobius tiptoni & 10 & 4 & $1: 0,40$ \\
Paratrichobius longicrus & 14 & 10 & $1: 0,71$ \\
Megistopoda aranea & 11 & 7 & $1: 0,64$ \\
M. proxima & 5 & 3 & $1: 0,6$ \\
Exastinion clovisi & 14 & 14 & $1: 1$ \\
Paraeuctenodes longipes & 4 & 3 & $1: 0,25$ \\
Anastrebla modestini & 12 & 4 & $1: 0,25$ \\
A. caudiferae & 7 & 5 & $1: 0,57$ \\
Metelasmus pseudopterus & 7 & & $1: 0,71$ \\
\hline
\end{tabular}

Em A. fimbriatus, M. pseudopterus foi a espécie encontrada em maior número de hospedeiros parasitados (tab. III) e a mais prevalente; no entanto, $M$. aranea foi a mais abundante (tab. IV). Em A. lituratus, P. longicrus foi encontrada em maior número de hospedeiros parasitados, foi mais prevalente e mais abundante (tabs. III, IV).

A comunidade de ectoparasitas sobre uma espécie de hospedeiro, em uma determinada área geográfica, pode ser tão característica, que poderia auxiliar na identificação do hospedeiro; no entanto, este padrão ocorreria apenas com parasitas nãomonoxenos (Wenzel et al., 1966:644). A composição da comunidade pode ser alterada por fatores climáticos e geográficos, comportamento do hospedeiro, disponibilidade das espécies de hospedeiros, relação histórica entre hospedeiro e parasita, entre outros (WENZEL et al., 1966; MARShall, 1982).

Tabela III. Número, freqüência relativa e número total de hospedeiros parasitados por uma ou mais espécies de Streblidae sobre Artibeus fimbriatus Gray e A. lituratus (Olfers) em Dom Pedro de Alcântara, Rio Grande do Sul, Brasil, durante 1997.

\begin{tabular}{|c|c|c|}
\hline Streblidae & $\begin{array}{l}\text { Artibeus fimbriatus } \\
\qquad(\mathrm{n}=23)\end{array}$ & $\begin{array}{c}\text { Artibeus lituratus } \\
(\mathrm{n}=37)\end{array}$ \\
\hline Paratrichobius longicrus & $(7,7 \%)$ & $(78,6 \%)$ \\
\hline Megistopoda aranea & $3(23,1 \%)$ & $(7,1 \%)$ \\
\hline Metelasmus pseudopterus & $6 \quad(46,2 \%)$ & 0 \\
\hline P. longicrus $+M$. aranea & $(7,7 \%)$ & $(14,3 \%)$ \\
\hline M. aranea + M. pseudopterus & $2 \quad(15,4 \%)$ & 0 \\
\hline Total de Indivíduos Parasitados & $13 \quad(100 \%)$ & $(100 \%)$ \\
\hline
\end{tabular}


Tabela IV. Prevalência (P) (\%) e abundância média (AM) com respectivos erro padrão de Paratrichobius longicrus (Miranda Ribeiro), Megistopoda aranea (Coquillett) e Metelasmus pseudopterus Coquillett sobre Artibeus fimbriatus Gray e A. lituratus (Olfers) em Dom Pedro de Alcântara, Rio Grande do Sul, Brasil, durante 1997.

\begin{tabular}{lcccc}
\hline \multirow{2}{*}{ Streblidae } & \multicolumn{2}{c}{ Artibeus fimbriatus } & \multicolumn{2}{c}{ Artibeus lituratus } \\
\cline { 2 - 5 } & $\mathrm{P}(\%)$ & $\mathrm{AM}$ & $\mathrm{P}(\%)$ & $\mathrm{AM}$ \\
\hline Paratrichobius longicrus & 8,7 & $0,17 \pm 0,806$ & 35,1 & $0,54 \pm 0,811$ \\
Megistopoda aranea & 21,7 & $0,65 \pm 1,312$ & 8,1 & $0,08 \pm 0,526$ \\
Metelasmus pseudopterus & 34,8 & $0,52 \pm 0,889$ & - & - \\
\hline
\end{tabular}

Os resultados indicam dois padrões característicos na composição das espécies de moscas ectoparasitas na área estudada sobre as espécies de Artibeus. Há uma comunidade característica sobre Artibeus fimbriatus, composta por Metelasmus pseudopterus e Megistopoda aranea; a ocorrência de P. longicrus seria apenas uma contaminação ou transitória, até o momento do encontro do hospedeiro preferencial. Sobre A. lituratus, ocorrem apenas Paratrichobius longicrus; a presença de qualquer outra espécie, como no caso de $A$. fimbriatus, poderia ser uma contaminação ou uma transição.

Agradecimento. A Hamilton C. Z. Grillo (UNIVATES / FATES), pelo auxílio na coleta do material na Furna BR 101, km 13/14.

\section{REFERÊNCIAS BIBLIOGRÁFICAS}

Autino, A. G.; Barquez, R. M. \& Claps, G. 1991. Nuevas citas de dipteros ectoparasitos (Streblidae) para murcielagos de la Argentina. Revta Soc. ent. argent., Buenos Aires, 50(1-4):248, 260.

Autino, A. G.; Claps, G. L. \& Bertolini, M. P. 1998. Primeros registros de insectos ectoparásitos (Diptera, Streblidae) de murciélagos de Parque Nacional Iguazú, Misiones, Argentina. Revta bras. Ent., São Paulo, 42(1/2):59-63.

Barquez, R. M.; Claps, G. \& Autino, A. G. 1991. Nuevos registros de ectoparasitos de murcielagos en el nororeste argentino. Revta Soc. ent. argent., Buenos Aires, 49(1-4):78, 102.

Bequeart, J. 1940. Moscas parásitas Pupiparas de Colombia y Panamá. Revta Acad. Colomb. Cienc. exact. fís. nat., Bogotá, 3(12):414-418.

1942. The Diptera Pupipara of Venezuela. Boln. Ent. venez., Caracas, 1(4):79-88.

Bush, A. O.; LafFerty, K. D. et al. 1997. Parasitology meets Ecology on it own terms: Margolis et al. revisited. J. Parasit., Lancaster, 83(4):575-583.

Coimbra, C. E. A.; Guimarães, L. R. \& Mello, D. A. 1984. Ocorrência de Streblidae (Diptera: Pupipara) em morcegos capturados em regiões de cerrado do Brasil Central. Revta bras. Ent., São Paulo, 28(4):547-550.

Guerrero, G. 1993. Catalogo de los Streblidae (Diptera: Pupipara) parasitos de murcielagos (Mammalia: Chiroptera) del Nuevo Mundo. I. Clave para los generos y Nycterophilinae. Acta biol. venez., Caracas, 14(4):61-75.

1994. Catalogo de los Streblidae (Diptera: Pupipara) parasitos de murcielagos (Mammalia: Chiroptera) del Nuevo Mundo. IV. Trichobiinae com alas desarrolladas. Boln Ent. venez., Nueva Serie, Caracas, 9(2):161-192.

1995a. Catalogo de los Streblidae (Diptera: Pupipara) parasitos de murcielagos (Mammalia: Chiroptera) del Nuevo Mundo. III. Los grupos: dugesii, dunni y phyllostomae del genero Trichobius Gervais, 1844. Acta biol. venez., Caracas, 15(3-4):1-27.

1995b. Catalogo de los Streblidae (Diptera: Pupipara) parasitos de murcielagos (Mammalia: Chiroptera) del Nuevo Mundo. V. Trichobiinae con alas reducidas o ausentes y miscelaneos. Boln Ent. venez., Nueva Serie, Caracas, 10(2):135-160. 
1996. Catalogo de los Streblidae (Diptera: Pupipara) parasitos de murcielagos (Mammalia:

Chiroptera) del Nuevo Mundo. VI. Streblinae. Acta biol. venez., Caracas, 16(2):1-26.

1997. Catalogo de los Streblidae (Diptera: Pupipara) parasitos de murcielagos (Mammalia:

Chiroptera) del Nuevo Mundo. VII. Lista de especies, hospedadores y paises. Acta biol. venez., Caracas, 17(1):9-24.

Guerrero, G. \& Morales-Malacara, J. B. 1996. Streblidae (Diptera: Calyptratae) parásitos de murciélagos (Mammalia: Chiroptera) cavernícolas del centro y sur de México, con descripción de una especie nueva del género Trichobius. An. Inst. Biol. Univ. Méx., Ser. Zool, México, 67(2):357-373.

Guimarães, L. R. 1941. Notas sobre Streblidae. Papéis Dep. Zool. S. Paulo, São Paulo, 1:213-222.

IBGE. 1986. Folha SH.22 Porto Alegre e parte das Folhas SH. 21 Uruguaiana e SI. 22 Lagoa Mirim: geologia, geomorfologia... Rio de Janeiro, IBGE. 796p. (Levantamento de recursos naturais, 33).

Jobling, B. 1949. Host-parasite relationship between the American Streblidae and the bats, with a new key to the american genera and a record of Streblidae from Trinidad, British West Indies (Diptera). Parasitology, Cambridge, 39:315-329.

Komeno, C. A. \& Linhares, A. X. 1999. Batflies parasitic on some phyllostomid bats in Southeastern Brazil: Parasitism rates and host-parasite relationships. Mems Inst. Oswaldo Cruz, Rio de Janeiro, 94(2): 151-156.

Marinkelle, C. J. \& Grose, E. S. 1981. A list of ectoparasites of Colombian bats. Revta Biol. trop., San José, 29(1):11-20.

Marshall, A. G. 1981. The sex ratio in ectoparasitic insects. Ecol. Ent., Oxford, 6:155-174.

1982. Ecology of insects ectoparasitic on bats. In: Kunz, T. H. ed. Ecology of bats. New York, Plenum. p. 369-401.

Miranda Ribeiro, A. 1907. Alguns dípteros interessantes. Arch. Mus. nac. Rio de J., Rio de Janeiro, 14:231-239.

Pessoa, S. B. \& Galvão, A. L. 1936. Novo genero e nova especie de Streblidae (Dipt. Pupipara) parasita do morcego do Brasil. Revta Ent., Rio de Janeiro, 6(2):242-248.

Pessoa, S. B. \& Guimarães, L. R. 1936. Notas sobre Streblidae (Diptera), com a descripção de um novo genero e duas novas especies. Anais Fac. Med. Univ. S. Paulo, São Paulo, 12(3):255-267.

1940. Nota sobre streblídeos (Diptera) de morcegos de Mato-Grosso, Brasil. Archos Inst. biol., São Paulo, 11:421-426.

Ross, A. 1961. Biological studies on bat ectoparasites of the Genus Trichobius (Diptera: Streblidae) in North America, north of Mexico. Wasmann J. Biol., San Francisco, 19:229-246.

Wenzel, R. L. 1970. A catalogue of the Diptera of the Americas south of the United States. Family Streblidae. São Paulo, Museu de Zoologia, Universidade de São Paulo, n.100, 25p.

1976. The streblid batflies of Venezuela (Diptera: Streblidae). Young Univ. Scien. Bull., Biol. Ser., Provo, 20(4):1-177.

Wenzel, R. L.; Tipton, V. J. \& Kiewlicz, A. 1966. The streblid batflies of Panama (Diptera: Calyptera: Streblidae). In: Wenzel, R. L. \& Tipton, V. J. eds. Ectoparasites of Panama. Chicago, Field Museum of Natural History. p. 405-675.

WhitAKER, J. O., JR. 1988. Collecting and preserving ectoparasites for ecological study. In: Kunz, T. H. ed. Ecological and Behavior Methods for the Study of Bats. Washington D. C., Smithsonian Institution. p. 459-474.

Whitaker, J. O., JR. \& Mumford, R. E. 1977. Records of ectoparasites from Brazilian mammals. Ent. News, Philadelphia, 88 (9-10): 255-258.

Recebido em 08.03.2000; aceito em 06.11.2000.

Iheringia, Sér. Zool., Porto Alegre, (90): 85-92, 25 de maio de 2001 